\begin{tabular}{|c|c|c|}
\hline Beitr. Ent. & Keltern & ISSN 0005-805X \\
\hline $\mathbf{5 5}(2005) 2$ & S. $313-318$ & 27.12 .2005 \\
\hline
\end{tabular}

\title{
Eine neue Gattung der Conopidae aus dem Baltischen Bernstein
}

\section{(Diptera: Conopidae)}

Mit 10 Figuren

Jens-Hermann Stuke

Zusammenfassung

Hoffeinsia baltica gen. nov. et spec. nov. wird aus dem Baltischen Bernstein (Eozän) beschrieben.

\section{Summary}

Hoffeinsia baltica gen. nov. et spec. nov. is described from the Baltic amber (Eocene).

Stichwörter

Diptera, Conopidae, Hoffeinsia, gen. nov., spec. nov., Baltischer Bernstein

keywords

Diptera, Conopidae, Hoffeinsia, gen. nov., spec. nov., Baltic amber

\section{Einleitung}

Nachdem bereits vor Kurzem ein Überblick über die Kenntnis der Blasenkopffliegen (Conopidae) des Baltischen Bernsteins gegeben wurde (STUKe 2003), konnten zwischenzeitlich zwei weitere Exemplare untersucht werden. Ein Exemplar (Baltischer Bernstein aus Jantarnyi, Kaliningrad, coll. HoffeIns [Nr. 1483.1]) lässt sich mit den bei STUKE (2003) genannten Merkmalen als Palaeomyopa tertiaria Meunier, 1912 bestimmen. Bei dem zweiten vorliegenden Exemplar handelt es sich um eine unbeschriebene Art, die gleichzeitig eine neue Gattung repräsentiert. Im Folgenden wird dieses Exemplar beschrieben. 


\title{
Gattung Hoffeinsia gen. nov.
}

\author{
Gattungstypus: Hoffeinsia baltica spec. nov.
}

Derivatio nominis: Die Gattung wird Christel und Hans Werner Hoffeins (Hamburg) gewidmet, die das hier bearbeitete Material aus ihrer umfangreichen Sammlung von Dipteren-Inklusen zur Verfügung stellten. Ihre freundschaftliche Hilfe bei der Arbeit mit Bernstein-Dipteren hat mir und vielen weiteren Dipterologen sehr geholfen.

\section{Beschreibung der Gattung (basierend auf einem Männchen):}

Fühlergruben sind nicht ausgebildet. Die Parafacialia nehmen jeweils etwa ein Drittel der Gesichtsbreite ein (Fig. 1). Der Rüssel ist kürzer als die Mundöffnung, und das Labellum ist stark erweitert (Fig. 4). Der Kopf trägt zwei Paar Orbitalborsten (or), ein Paar Postvertikalborsten (pvt), ein Paar innere Scheitelborsten (vti) und ein Paar äußere Scheitelborsten (1 vte) (Fig. 3). Auf dem Thorax befinden sich jeweils eine Humeralborste $(1 \mathrm{~h}), 2$ Notopleuralborsten $(2 \mathrm{n})$, eine Supraalarborste (sa), zwei Postalarborsten (pa), vier Borstenpaare zwischen Quernaht und Scutellum, bei denen es sich um Dorsozentralborsten (dc) und Praescutellarborsten (psc) handeln wird, und zwei Paar Scutellarborsten (sc). Katepisternum und Propleuron tragen keine Borsten. Das Gesicht ist medial nicht längs gekielt und im Profil konkav (Fig. 2). Das Femur (am auffälligsten das Hinterfemur, Fig. 5) sind verdickt und vollständig behaart. Auf den Coxen kommen keine Borsten vor. Der Trochanter des 3. Beines trägt zwei auffällige Ausstülpungen, und zwar anterior eine dreieckige kielartige Ausstülpung und posterior eine schmale zahnartige Ausstülpung. Beide Ausstülpungen sind mit kurzen, kräftigen schwarzen Haaren besetzt (Fig. 6). Tergite 5-7 sind jeweils dorsal verlängert, und das Abdomen ist daher insgesamt nach ventral umgebogen (Fig. 9).

\section{Diagnose:}

Hoffeinsia ähnelt der ebenfalls nur aus Baltischem Bernstein bekannten Gattung Palaeomyopa Meunier, 1899 durch (a) das Fehlen der Fühlergruben, (b) hinsichtlich der breiten Parafacialia, (c) auf Grund des kurzen Rüssels mit seinen apikal erweiterten Labellen und (d) hinsichtlich der Anordnung der Borsten auf Kopf und Mesoscutum. Durch die Kombination dieser Merkmale unterscheiden sich die beiden Gattungen von allen rezenten Conopiden. Hoffeinsia hebt sich von Palaeomyopa durch mehrere Merkmale ab: (a) das ungekielte und im Profil konkave Gesicht, (b) das Fehlen eines dritten Paares Orbitalborsten sowie das Fehlen von Borsten auf dem Katepisternum, dem Propleuron und den Coxen, (c) die verdickten und vollständig behaarten Femora, (d) die charakteristischen Ausstülpungen auf den hinteren Trochantern und (e) die dorsal verlängerten Tergite 5-7 und das daraus resultierende umgebogene Abdomenende.

\section{Diskussion der Phylogenie:}

Die Gattung Hoffeinsia kann nicht befriedigend in das System der Conopidae eingeordnet werden: Bereits Hennig (1966) weist darauf hin, dass die ähnliche Gattung Palaeomyopa nach damaligem Kenntnisstand zweifellos nicht den Stylogasterinae aber auch nicht den Conopinae oder den Myopinae zugeordnet werden kann. Da alle von Hennig (l. c.) für Palaeomyopa diskutierten Merkmale auch für Hoffeinsia zutreffen, können seine Überlegungen für die neue Gattung übernommen werden. CAMras (1994) 
stellt eine eigene Unterfamilie - die Palaeomyopinae - auf und ordnet nur die Gattung Palaeomyopa hier ein. Da er die neue Unterfamilie aber mit keiner überzeugenden Apomorphie begründet (Diskussion in STUKE 2003), ist seine Neubeschreibung wenig hilfreich, um die Einordnung von Hoffeinsia zu diskutieren. Ein Schwestergruppenverhältnis von Hoffeinsia und Palaeomyopa kann ohne kladistische Analyse nicht belegt werden, da die Ähnlichkeit der beiden Gattungen auf Merkmalen beruht, die entweder Plesiomorphien sind oder die nicht als plesiomorph oder apomorph gewertet werden können. Synapomorphien, die ein Schwestergruppenverhältnis von Hoffeinsia und Palaeomyopa begründen, wurden nicht gefunden.

\section{Hoffeinsia baltica spec. nov.}

(Fig. 1-10)

Holotypus: Männchen in Baltischem Bernstein (Eozän), Jantarnyi, Samland Halbinsel, Kaliningrad Region, Privatsammlung Hoffeins, Nr. 1483/2. Der Typus soll später der Sammlung des Deutschen Entomologischen Instituts, Müncheberg [DEI], übergeben werden.

Erhaltung: Die Fühler, die linke Vordertibia und die linken Hintertarsen sind teilweise abgeschliffen. Der umgebende Bernstein enthält mehrere kleine Verschmutzungen. Darüberhinaus ist das Exemplar gut erhalten. Syninklusen kommen nicht vor. Das Präparat ist in Kunstharz (GTS Polyester-Gießharz der Firma Vosschemie) eingegossen.

Derivatio nominis: Der Artname beschreibt die Herkunft des Typus aus dem östlichen Ostseeraum.

Diagnose: Die Art kann mit den in der Gattungsdiagnose genannten Merkmalen bestimmt werden.

\section{Beschreibung des Holotypus ( $\left.o^{\star}\right)$}

Kopf: Scapus und Pedicellus behaart. Vom Postpedicellus ist nur auf einer Seite ein schmaler angeschliffener Rest übrig, die Arista ist nicht mehr vorhanden. Form der Fühler entsprechend Fig. 8. Facettenaugen unbehaart, Facetten gleich groß. Ocellenhöcker schwach ausgeprägt, mit drei deutlich ausgebildeten Ocellen und einem Paar Ocellarborsten (oc). Ocellendreieck schmal und lang, entsprechend Fig. 3. Stirn mit zwei Paar Orbitalborsten (or). Stirnbehaarung deutlich länger als Ocellendurchmesser und die ganze Stirn, ausgenommen das Ocellendreieck, bedeckend. Gesicht nicht gekielt, ohne Fühlergruben, mit breiten und deutlich abgesetzten Parafacialia. Gesichtsprofil konkav. Unterseite des Gesichtes behaart und zusätzlich einzelne Haare an der inneren Grenze der Parafacialia. Postcranium mit Ausnahme des Occipitalsklerites mit langer, dichter, abstehender Behaarung, die nach ventral nicht auffällig länger wird; mit jeweils einem Paar Postvertikalborsten (pvt), inneren Scheitelborsten (vti) und äußeren Scheitelborsten (vte). Rüssel entsprechend Fig. 4: insgesamt kürzer als die Mundöffnung, Labellen erweitert. Palpen länger als Pedicellus, lateral lang behaart (etwa doppelte Länge der Palpenbreite). 

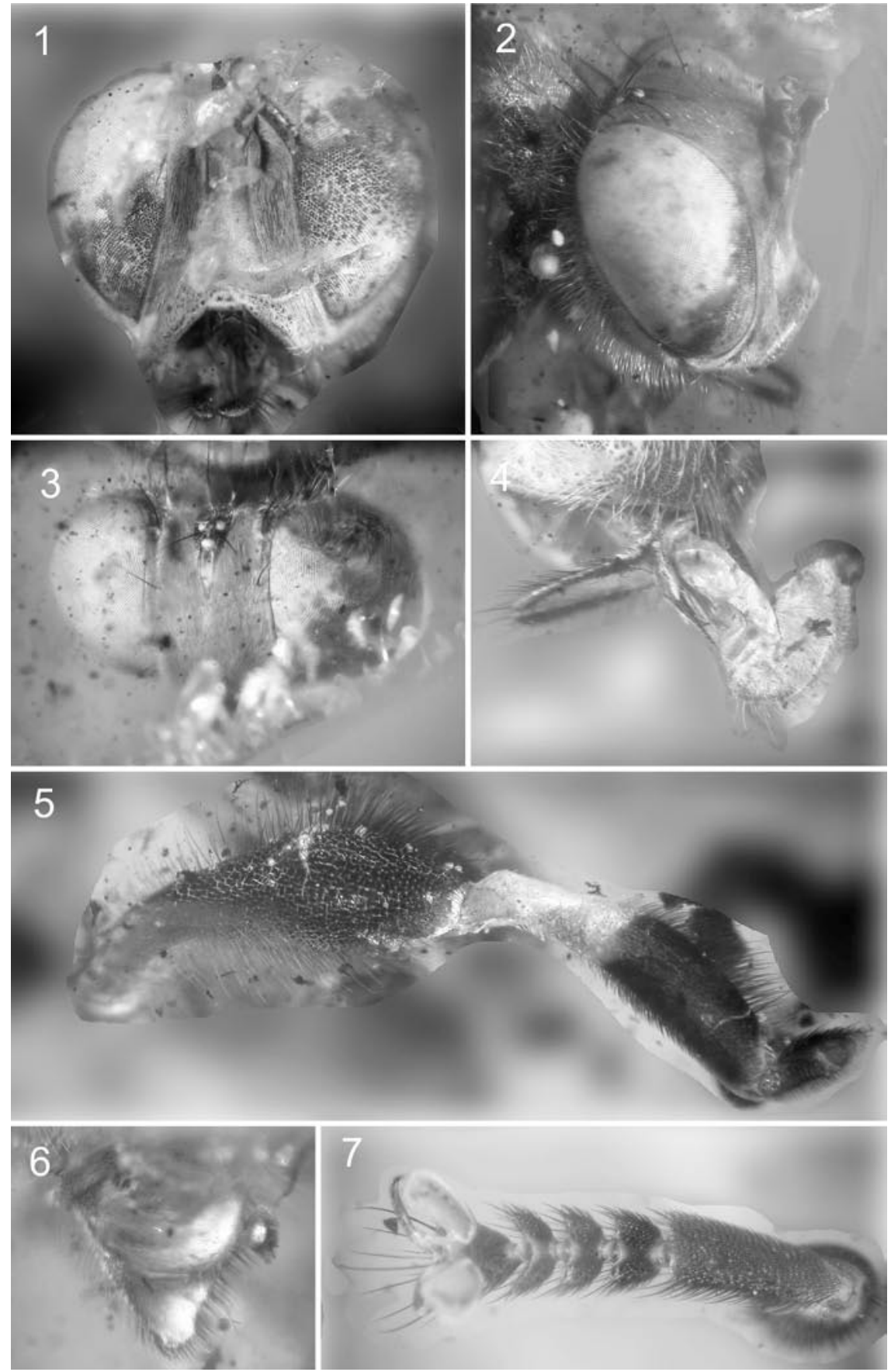

Fig. 1-7: Hoffeinsia baltica gen. nov. et spec. nov. [Holotypus] - 1: Kopf frontal; 2: Kopf dorsolateral; -3: Kopf dorsal; - 4: Rüssel lateral; - 5: Hinterbein; - 6: Hintercoxa; - 7: Vordertarsen ventral. 

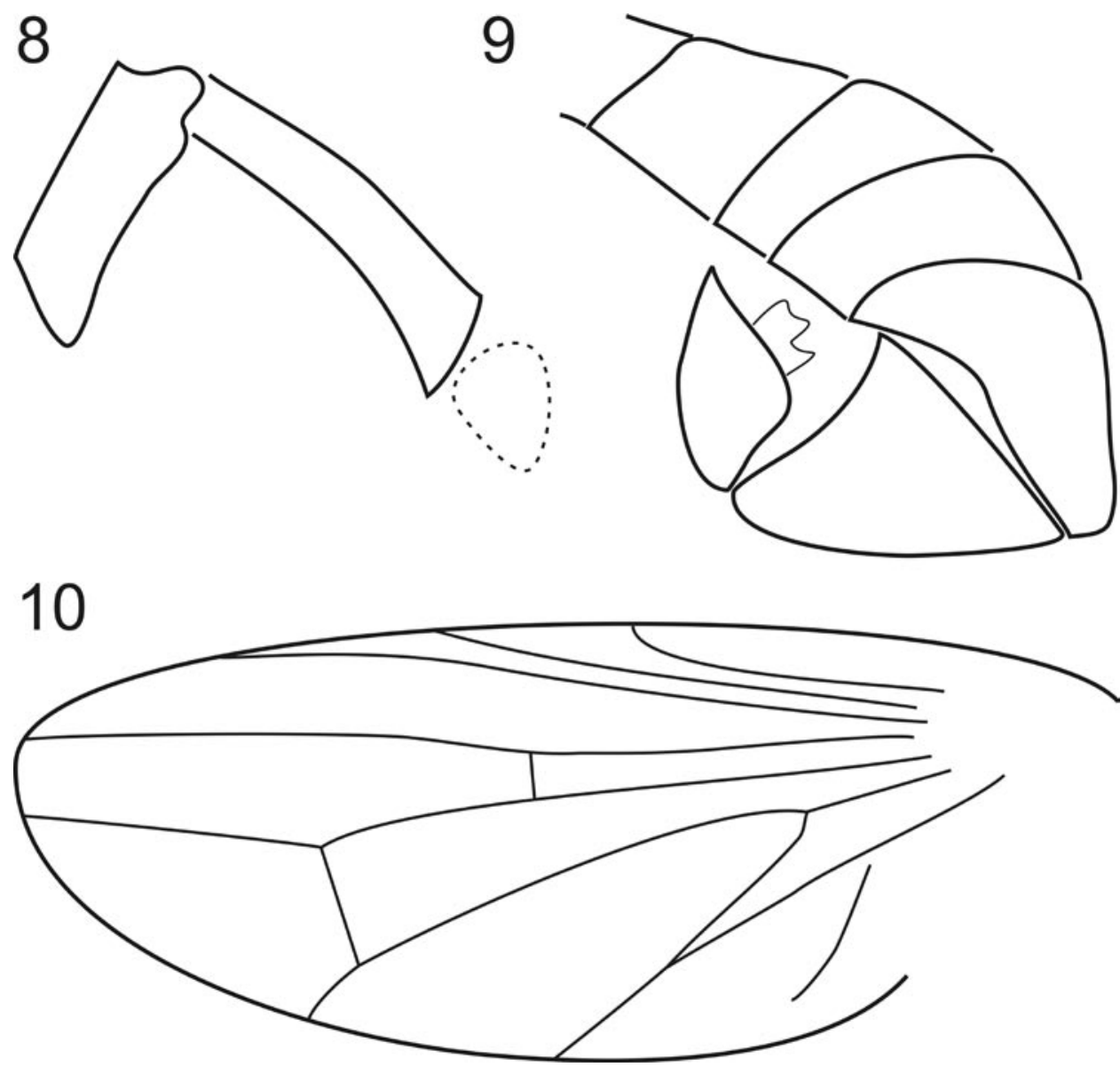

Fig. 8-10: Hoffeinsia baltica gen. nov. et spec. nov. [Holotypus] - 8: Fühler [Rekonstruktion aus beiden Fühlerresten; der gestrichelt eingezeichnete Postpedicellus ist nur im Querschnitt zu erkennen]; - 9: Abdomen mit Surstylus; - 10: Flügeladerung.

Thorax: Mesoscutum vollständig behaart, mit charakteristischer Beborstung: Jeweils eine Humeralborste (h), 2 Notopleuralborsten (n), eine Supraalarborste (sa), zwei Postalarborsten (pa) und vier Borstenpaare zwischen Thorakalquernaht und Scutellum, bei denen es sich um Dorsozentralborsten (dc) und Praescutellarborsten (psc) handeln dürfte. Scutellum vollständig behaart, am Hinterrand mit zwei Paar lateralen Scutellarborsten (sc). Weitere Borsten kommen auf dem Thorax nicht vor, insbesondere fehlen sie auf dem Katepisternum und dem Propleuron. Eine lange Behaarung findet man auf der hinteren Hälfte des Katepisternums und auf dem Propleuron. Flügellänge 8,3 mm. Flügel vollständig mit Mikrotrichien bedeckt. Flügeladerung entsprechend Fig. 10: Radialzelle $\mathrm{r}_{4+5}$ offen; Analzelle cup geschlossen; Ader CuA 2 basal schwach geknickt; Subcosta und Radialader $\mathrm{R}_{1}$ deutlich getrennt. Auf den Halteren keine Borsten nachweisbar. Calyptra am Rand mit langen Haaren. 
Femur und Tibia auffällig verdickt, besonders das Hinterfemur erreicht eine für Conopiden einzigartige Form (Fig. 5). Beine vollständig dicht behaart, besonders lang ist die dorsale und ventrale Behaarung auf den Femur und die dorsale Behaarung auf den Tibia. Tibien mit dorsaler Praeapikalborste. Tibien im basalen Drittel scharf abgesetzt weiß, apikal dunkelbraun. Femur dunkelbraun und davon abgesetzt basal hellbraun, die hellbraune Färbung ist auf der Ventralseite weiter ausgedehnt. Coxen behaart aber ohne Borsten. Der hintere Trochanter mit zwei auffälligen Ausstülpungen, anterior eine dreieckige kielartige Ausstülpung und posterior eine schmale zahnartige Ausstülpung; beide Ausstülpungen mit kurzen, kräftigen schwarzen Haaren besetzt (Fig. 6). Tarsen dunkel, dorsal und lateral mit einzelnen Borsten, ventral mit dichter hellgelber Behaarung. Klauen basal hell und apikal schwarz. Pulvillen und Empodiumweiß. Form der Tarsenglieder entsprechend Fig. 7.

Abdomen: Abdomen vollständig behaart. Tergit 5-7 dorsal verlängert, die Abdomenspitze umgebogen (Fig. 9). Surstylus bei Tergit 7 erkennbar, seine Form entsprechend Fig. 9.

\title{
Danksagung
}

Für das Ausleihen von Material danke ich Christel und Hans Werner Hoffeins (Hamburg). Hinweise zu früheren Versionen des Manuskriptes verdanke ich Claus Claußen (Flensburg), Fritz Geller Grimm (Frankfurt), Martin Hauser (Champaign), Dr. Frank Menzel (Müncheberg) und Dr. Michael von Tschirnhaus (Bielefeld).

\section{Literatur}

Camras, S. 1994: A new subfamily for the fossil Conopid fly Palaeomyopa tertiaria (Diptera: Conopidae). - Entomological News 105: 175-177; Philadelphia.

Hennig, W. 1966: Conopidae im Baltischen Bernstein (Diptera, Cyclorrhapha). - Stuttgarter Beiträge zur Naturkunde 154: 1-24; Stuttgart.

Stuke, J.-H. 2003: Eine neue Blasenkopffliege der Gattung Palaeomyopa Meunier aus dem Baltischen Bernstein (Diptera: Syrphidae). - Studia dipterologica 10: 91-96; Halle.

\author{
Anschrift des Verfassers: \\ Dr. Jens-Hermann Stuke \\ Brunnenstraße 28 \\ D-26789 Leer \\ jstuke@zfn.uni-bremen.de
}

